\title{
Cold Dark Matter Substructure and Galactic Disks
}

\author{
Stelios Kazantzidis ${ }^{1}$, Andrew R. Zentner ${ }^{2}$, and James S. Bullock ${ }^{3}$ \\ ${ }^{1}$ Center for Cosmology and Astro-Particle Physics, The Ohio State University, \\ 191 West Woodruff Avenue, Columbus, OH 43210, USA \\ email: stelios@mps.ohio-state.edu \\ ${ }^{2}$ Department of Physics \& Astronomy, University of Pittsburgh, \\ 100 Allen Hall, 3941 O'Hara Street, Pittsburgh, PA 15260, USA \\ email: zentner@pitt.edu \\ ${ }^{3}$ Center for Cosmology, Department of Physics \& Astronomy, \\ The University of California at Irvine, 4168 Reines Hall, Irvine, CA 92697, USA \\ email: bullock@uci.edu
}

\begin{abstract}
We perform a set of high-resolution, fully self-consistent dissipationless $N$-body simulations to investigate the influence of cold dark matter (CDM) substructure on the dynamical evolution of thin galactic disks. Our method combines cosmological simulations of galaxy-sized $\mathrm{CDM}$ halos to derive the properties of substructure populations and controlled numerical experiments of consecutive subhalo impacts onto initially-thin, fully-formed disk galaxies. We demonstrate that close encounters between massive subhalos and galactic disks since $z \sim 1$ should be common occurrences in $\Lambda$ CDM models. In contrast, extremely few satellites in present-day CDM halos are likely to have a significant impact on the disk structure. One typical host halo merger history is used to seed controlled $N$-body experiments of subhalo-disk encounters. As a result of these accretion events, the disk thickens considerably at all radii with the disk scale height increasing in excess of a factor of 2 in the solar neighborhood. We show that interactions with the subhalo population produce a wealth of distinctive morphological signatures in the disk stars, many of which resemble those being discovered in the Milky Way (MW), M31, and in other disk galaxies, including: conspicuous flares; bars; low-lived, ring-like features in the outskirts; and low-density, filamentary structures above the disk plane. These findings highlight the significant role of CDM substructure in setting the structure of disk galaxies and driving galaxy evolution.
\end{abstract}

Keywords. cosmology: theory, dark matter, galaxies: formation, galaxies: dynamics, galaxies: structure, methods: numerical

\section{Introduction}

The currently favored cold dark matter (CDM) paradigm of hierarchical structure formation (e.g., Blumenthal et al. 1984), predicts significant dark matter halo substructure in the form of small, dense, self-bound subhalos orbiting within the virialized regions of larger host halos (e.g., Klypin et al. 1999). Observational probes of substructure abundance thus constitute fundamental tests of the CDM model. Due to the fact that most subhalos associated with galaxy-sized host halos lack of a significant luminous component, a constraint on the amount of substructure in these systems may be obtained via their gravitational influence on galactic disks. If there is a considerable subhalo population, it may produce strong tidal effects and induce distinctive gravitational signatures which might be imprinted on the structure and kinematics of the host galactic disk. Thus, establishing the role of substructure in shaping the fine structure of galactic disks may 
prove fundamental in informing our ideas about global properties of galaxy formation and evolution.

Significant theoretical effort has been devoted to quantifying the resilience of galactic disks to infalling satellites (e.g., Quinn \& Goodman 1986; Velazquez \& White 1999; Font et al. 2001; Gauthier et al. 2006; Read et al. 2008; Villalobos \& Helmi 2008). Despite their usefulness, most earlier investigations suffered basic shortcomings that limited their applicability. For example, some considered encounters of single satellites with galactic disks, a set-up which is at odds with CDM predictions of multiple, nearly contemporaneous accretion events. Other studies made ad hoc assumptions about the orbital parameters and internal structures of the infalling systems. Consequently, it remains uncertain whether these earlier investigations faithfully captured the responses of galactic disks to halo substructure in a cosmological context. Here we address this issue using a hybrid approach that combines cosmological simulations to derive the merger histories of galaxy-sized CDM halos with controlled numerical experiments of consecutive subhalo impacts onto $N$-body realizations of fully-formed disk galaxies.

\section{Methods}

A thorough description of our methods is presented in Kazantzidis et al. (2007) and we summarize them here. First, we analyze cosmological simulations of the formation of four galaxy-sized halos in the $\Lambda$ CDM cosmology. The simulations were performed with the Adaptive Refinement Tree (ART) N-body code (Kravtsov 1999). All of these halos accrete only a small fraction of their final masses and experience no major mergers at $z \lesssim 1$ (a look-back time of $\approx 8 \mathrm{Gyr}$ ), and therefore may reasonably host a disk galaxy. Second, we identify subhalos in these hosts and select the massive substructures that pass near the center of the host halo where they may interact appreciably with a galactic disk for further consideration. Finally, we use a representative subset of these accretion events from one of the host halos to seed controlled $N$-body simulations of satellite impacts onto an initially-thin disk galaxy.

While the present work is informed by many past numerical investigations of satellitedisk interactions, our methodology is characterized by at least three major improvements. First, we consider satellite populations whose properties are extracted directly from the cosmological simulations of galaxy-sized CDM halos. This eliminates many assumptions regarding the internal properties and impact parameters of infalling systems inherent in many previous studies. Second, we employ primary disk galaxy models that are both selfconsistent and flexible enough to permit detailed modeling of actual galaxies such as the MW and M31 by fitting to a wide range of observational data sets (Widrow \& Dubinski 2005). In this work, we employ galaxy model MWb of Widrow \& Dubinski (2005) which reproduces many of the observed characteristics of the MW galaxy. This galaxy model comprises an exponential stellar disk with a $\operatorname{sech}^{2}$ scale height of $z_{d}=400 \mathrm{pc}$, a Hernquist model bulge, and an NFW dark matter halo.

Lastly, and most importantly, we incorporate for the first time a model in which the infalling subhalo populations are representative of those that impinge upon halo centers since $z \sim 1$, instead of the $z=0$ surviving substructure present in a CDM halo. Previous studies utilized the present-day properties of a large ensemble of dark matter subhalos in order to investigate the dynamical effects of substructure on galactic disks (Font et al. 2001; Gauthier et al. 2006). Successes notwithstanding, this methodology has the drawback of eliminating from consideration those massive satellites that, prior to $z=0$, pass very close to the central regions of their hosts, where the galactic disk resides. These systems can potentially produce strong tidal effects on the disk, but are 


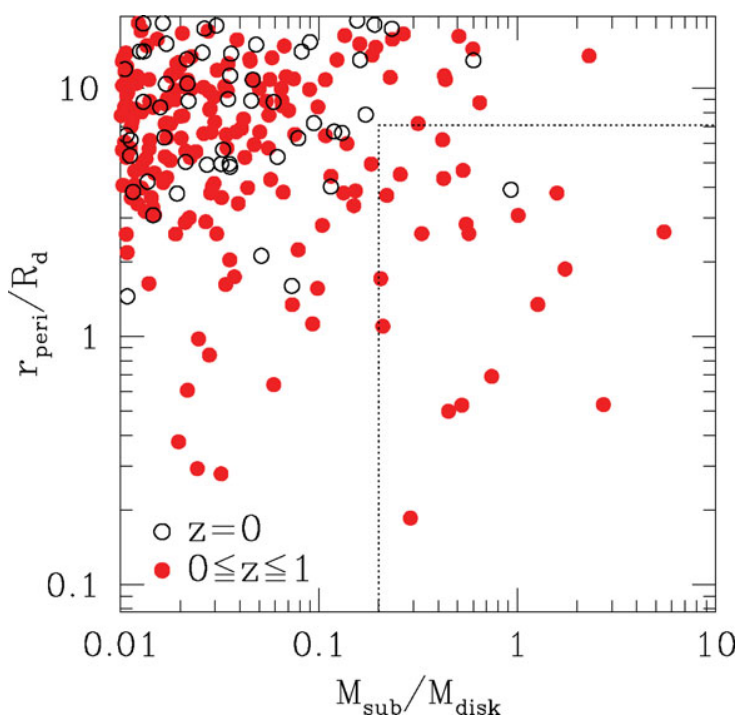

Figure 1. A scatter plot of mass versus pericentric distance for satellites identified in four galaxy-sized halos formed in the $\Lambda$ CDM cosmology. Subhalo masses and orbital radii are presented in units of the mass, $M_{\mathrm{disk}}=3.53 \times 10^{10} \mathrm{M}_{\odot}$, and radial scale length, $R_{d}=2.82 \mathrm{kpc}$, respectively, of the galactic disk in the controlled simulations. Filled symbols show results for subhalos that pass closer than an infall radius of $r_{\mathrm{inf}}=50 \mathrm{kpc}$ of their host halo center since a redshift $z=1$. Open symbols refer to the $z=0$ population of surviving substructures. Accretions of massive subhalos onto the central regions of their hosts, where the galactic disk resides, since $z \sim 1$ should be common occurrences in standard $\Lambda$ CDM.

unlikely to constitute effective perturbers at $z=0$ as they suffer substantial mass loss during their orbital evolution precisely because of their forays into the central halo (e.g., Zentner \& Bullock 2003).

Figure 1 illustrates the importance of accounting for subhalo infall over time. This figure is a scatter plot of mass versus pericentric distance for two different satellite populations within all four galaxy-sized host CDM halos. The masses and distances in Figure 1 have been scaled to the mass, $M_{\text {disk }}=3.53 \times 10^{10} \mathrm{M}_{\odot}$, and radial scale length, $R_{d}=2.82 \mathrm{kpc}$, of the stellar disk in the primary galaxy model used in the satellite-disk encounter simulations. The dotted line encloses an area in the $M_{\text {sub }}-r_{\text {peri }}$ plane corresponding to subhalos more massive than $0.2 M_{\text {disk }}$ with pericenters of $r_{\text {peri }} \lesssim 20 \mathrm{kpc}$ $\left(r_{\text {peri }} \lesssim 7 R_{d}\right)$. We refer to this area as the "danger zone". Satellites within this area are expected to constitute effective perturbers and may cause considerable damage to the disk, but we intend this as a rough criterion to aid in illustrating our point.

The first satellite population in Figure 1 consists of the $z=0$ surviving substructures. The second subhalo population consists of systems that approach the central regions of their hosts since a redshift $z=1$. These subhalos cross within a (scaled) infall radius of $r_{\text {inf }}=50 \mathrm{kpc}$ from the host halo center. This selection is fixed empirically to identify orbiting substructure that are likely to have a significant dynamical impact on the structure of the disk (Kazantzidis et al. 2007). The masses associated with this group of satellites are defined at the simulation output time nearest the inward crossing of $r_{\text {inf }}$. Pericenters are computed from the orbit of a test particle in a static NFW potential whose properties match those of the host CDM halo at the time of $r_{\text {inf }}$.

Figure 1 demonstrates that the $z=0$ subhalo populations contain very few massive systems on potentially damaging orbits. In fact, statistics of all four galaxy-sized host halos indicate that only one satellite can be identified inside the danger zone in this 
case. On the other hand, the danger zone contains numerous substructures that passed through or near the galactic disk since $z=1$. On average, $\sim 5$ satellites more massive than $0.2 M_{\text {disk }}$ cross through the central region of a galaxy-sized halo with $r_{\text {peri }} \lesssim 20 \mathrm{kpc}$ during this period. This suggests that close encounters between massive subhalos and galactic disks since $z=1$ are common occurrences in standard $\Lambda$ CDM. Thus, it is important to account for such accretion events to model the cumulative dynamical effects of halo substructure on disk galaxies.

In what follows, we focus on one of the host halo accretion histories to seed controlled $N$-body experiments of subhalo-disk encounters. We identify target satellites that are likely to have a substantial effect on the disk structure by imposing two selection criteria. First, we limit our search to satellites that approach the central region of their host with small orbital pericenters $\left(r_{\text {peri }} \lesssim 20 \mathrm{kpc}\right)$ since $z=1$. Second, we restrict re-simulation to subhalos that are a significant fraction of the disk mass, but not more massive than the disk itself $\left(0.2 M_{\text {disk }} \lesssim M_{\text {sub }} \lesssim M_{\text {disk }}\right)$. The aforementioned criteria resulted in six accretion events of satellites with masses and tidal radii of $7.4 \times 10^{9} \lesssim M_{\text {sub }} / M_{\odot} \lesssim$ $2 \times 10^{10}$, and $r_{\text {tid }} \gtrsim 20 \mathrm{kpc}$, respectively, from a single host to simulate over a $\sim 8 \mathrm{Gyr}$ period. Additional properties of these substructures can be found in Kazantzidis et al. (2007). We modeled subhalo impacts onto the disk as a sequence of encounters. Starting with the first satellite, we included subsequent systems at the epoch when they were recorded in the cosmological simulation.

We extracted the density structures of these cosmological subhalos and followed the procedure outlined in Kazantzidis et al. (2004) to construct self-consistent, $N$-body realizations of satellites models. Each system was represented with $N_{\text {sat }}=10^{6}$ particles and a gravitational softening length of $\epsilon_{\mathrm{sat}}=150 \mathrm{pc}$. For the primary disk galaxy, we used $N_{d}=10^{6}$ particles to represent the disk, $N_{b}=5 \times 10^{5}$ in the bulge, and $N_{h}=2 \times 10^{6}$ in the dark matter halo, and softenings of $\epsilon_{d}=50 \mathrm{pc}, \epsilon_{b}=50 \mathrm{pc}$, and $\epsilon_{h}=100 \mathrm{pc}$, respectively. All satellite-disk encounter simulations were carried out using PKDGRAV (Stadel 2001). The "final" disk discussed in the next sections has experienced all six subhalo impacts and was evolved in isolation for $\sim 4$ Gyr after the last interaction. Finally, we compute all disk properties and show all visualizations of the disk morphology after centering the disk to its center of mass and rotating it to a new coordinate frame defined by the three principal axes of the total disk inertia tensor.

\section{Global Disk Morphology}

Figure 2 depicts the transformation of the global structure of a thin galactic disk that experiences a merging history of the kind expected in the $\Lambda$ CDM paradigm of structure formation. This figure shows face-on and edge-on views of the initial and final distribution of disk stars.

Figure 2 demonstrates that encounters with CDM substructure are responsible for generating several distinctive morphological signatures in the disk. The final disk is considerably thicker (or "flared") compared to the initial distribution of disk stars and a wealth of low-density features have developed both in and above the disk plane as a consequence of these disturbances. Particularly intriguing is the fact that a high-density, in-plane structure survives after the satellite bombardment. A standard "thin-thick" disk decomposition analysis for the final disk indicates that this feature would be recognized as a thin disk component (Kazantzidis et al. 2007). The edge-on view of the final disk also reveals additional filamentary structures and other complex configurations above the disk plane. These structures bear some resemblance to tidal streams, but are in fact disk stars that have been excited by the subhalo impacts. Interestingly, the same image shows 


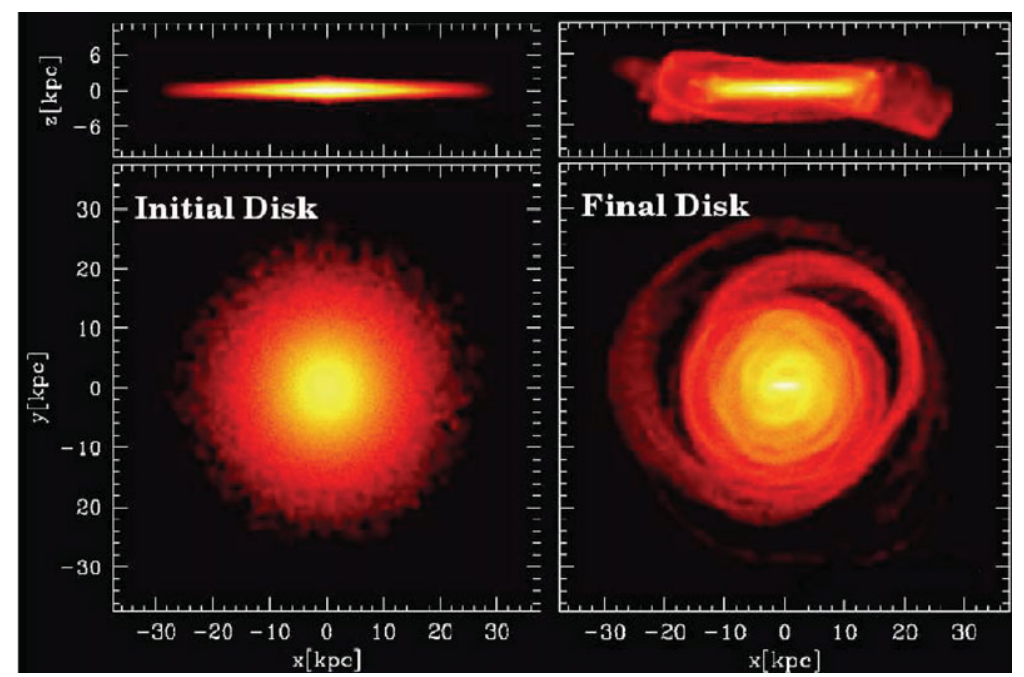

Figure 2. Density maps of disk stars illustrating the global morphological transformation of a galactic disk subject to a $\Lambda$ CDM-motivated satellite accretion history. The left panel shows the initial disk assuming that the sequence of satellite-disk interactions initiates at $z=1$, while the right panel depicts the disk after the last satellite passage, evolved in isolation for additional $\sim 4 \mathrm{Gyr}$, so that the evolution of disk stars is followed from $z=1$ to $z=0$. The edge-on (upper panels) and face-on (bottom panels) views of the disk are displayed in each frame. Satellite-disk interactions of the kind expected in $\Lambda \mathrm{CDM}$ models produce several distinctive signatures in galactic disks including: long-lived, low-surface brightness, ring-like features in the outskirts; conspicuous flares; bars; and faint filamentary structures above the disk plane that (spuriously) resemble tidal streams in configuration space. These morphological features are similar to those being discovered in the Milky Way, M31, and in other disk galaxies.

a characteristic " $\mathrm{X}$ " shape in the bright central disk, a finding also reported by Gauthier et al. (2006). This feature is often linked to secular evolution of galaxies driven by the presence of a bar when it buckles as a result of becoming unstable to bending modes.

The face-on image of the final disk illustrates the formation of a moderately strong bar and extended ring-like features in the outskirts of the disk. The existence of these features indicate that the axisymmetry of the disk has been destroyed by the encounters with the infalling subhalos. We emphasize that the aforementioned structures are persistent, surviving for a considerable time after the satellite passages ( $\sim 4 \mathrm{Gyr})$, and that the bar is induced in response to the accretion events, not by amplified noise.

\section{Disk Thickening}

Among the most striking signatures induced by the subhalo accretion events in our simulations is the pronounced increase in disk thickness. A quantitative analysis of disk thickening is presented in Figure 3. This figure shows that the initial disk thickens considerably at all radii as a result of the substructure impacts. Remarkably, the scale height of the disk near the solar radius increases in excess of a factor of 2 . The outer disk is much more susceptible to damage by the infalling satellites: at $R=R_{d}$ the scale height grows by $\sim 50 \%$ compared to approximately a factor of 3 increase at $R=4 R_{d}$. The larger binding energy of the inner, exponential disk and the presence of a massive bulge $\left(M_{b} \sim 0.3 M_{\text {disk }}\right)$ that acts as a sink of satellite orbital energy are responsible for the robustness of the inner disk. 


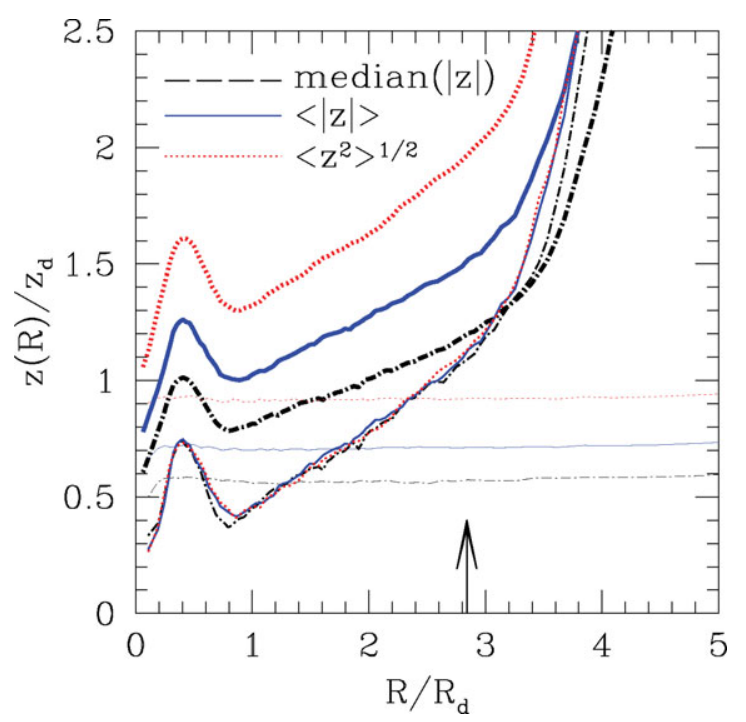

Figure 3. Disk thickening. Thickness profiles, $z(R)$, for the disk initially (thin lines) and after the satellite passages (thick lines). Lines of intermediate thickness show the fractional increase in disk thickness caused by the subhalo bombardment. Profiles are normalized to the initial disk scale height, $z_{d}$, and are plotted as a function of projected radius in units of the disk radial scale length, $R_{d}$. Different lines correspond to different measures of disk thickness. The arrow indicates the location of the solar radius, $R_{\odot}$. The galactic disk thickens considerably at all radii as a result of the encounters with CDM substructure.

Font et al. (2001) and Gauthier et al. (2006) performed similar numerical studies of the dynamical response of disks to CDM subhalos. Both investigations reported negligible tidal effects on the global structure of the disk. In contrast, Figure 3 indicates substantial disk thickening due to substructure bombardment. The primary reason for this discrepancy is that we followed the formation history of a host halo since $z \sim 1$, whereas Font et al. (2001) and Gauthier et al. (2006) considered the $z=0$ population of surviving substructure present in a CDM halo.

\section{References}

Blumenthal, G. R., Faber, S. M., Primack, J. R., \& Rees, M. J. 1984, Nature, 311, 517

Font, A. S., Navarro, J. F., Stadel, J., \& Quinn, T. 2001, Ap. Lett., 563, L1

Gauthier, J.-R., Dubinski, J., \& Widrow, L. M. 2006, ApJ, 653, 1180

Kazantzidis, S., Bullock, J. S., Zentner, A. R., Kravtsov, A. V., \& Moustakas, L. A. 2007, ApJ accepted (astro-ph/0708.1949)

Kazantzidis, S., Magorrian, J. \& Moore, B. 2004, ApJ, 601, 37

Klypin, A., Kravtsov, A. V., Valenzuela, O., \& Prada, F. 1999, ApJ, 522, 82

Kravtsov, A. V. 1999, PhD thesis, New Mexico State University

Quinn, P. J. \& Goodman, J. 1986, ApJ, 309, 472

Read, J. I., Lake, G., Agertz, O., \& Debattista, V. P. 2008, MNRAS accepted (astro$\mathrm{ph} / 0803.2714)$

Stadel, J. G. 2001, Ph.D. Thesis, Univ. of Washington

Velazquez, H. \& White, S. D. M. 1999, MNRAS, 304, 254

Villalobos, Á. \& Helmi, A. 2008, MNRAS submitted (astro-ph/0803.2323)

Widrow, L. M. \& Dubinski, J. 2005, ApJ, 631, 838

Zentner, A. R. \& Bullock, J. S. 2003, ApJ, 598, 49 